\title{
Expression, refolding and spectroscopic characterization of Fibronectin type III (FnIII)-homology domains derived from human Fibronectin Leucine Rich Transmembrane Protein (FLRT)-1, -2, and -3
}

Lila Yang ${ }^{1}$, Maria Hansen Falkesgaard ${ }^{2}$ ， Peter W Thulstrup ${ }^{1}$, Peter S Walmod ${ }^{2}$, Leila Lo Leggio ${ }^{\text {Corresp., }}{ }^{1}$, Kim Krighaar Rasmussen ${ }^{\text {Corresp. } 1,2}$

1 Biological Chemistry, Department of Chemistry, University of Copenhagen, Copenhagen, Denmark

2 Laboratory of Neural Plasticity, Department of Neuroscience and Pharmacology, Faculty of Health and Medical Sciences, University of Copenhagen, Copenhagen, Denmark

Corresponding Authors: Leila Lo Leggio, Kim Krighaar Rasmussen

Email address: leila@chem.ku.dk, KKR@chem.ku.dk

The fibronectin leucine rich transmembrane (FLRT) protein family consists in humans of 3 proteins, FLRT1, -2 , and -3 . The FLRT proteins contain 2 extracellular domains separated by an unstructured linker. The most membrane distal part is a leucine rich repeat (LRR) domain responsible for both cis- and trans-interactions, whereas the membrane proximal part is a fibronectin type III (FnIII) domain responsible for a cis-interaction with members of the fibroblast growth factor receptor 1 (FGFR1) family, which results in FGFR tyrosine kinase activation. Whereas the structures of FLRT LRR domains from various species have been determined, the expression and purification of recombinant FLRT FnIII domains, important steps for further structural and functional characterizations of the proteins, have not yet been described. Here we present a protocol for expressing recombinant FLRT-FnIII domains in inclusion bodies in Escherichia coli. His-tags permitted affinity purification of the domains, which subsequently were refolded on a Ni-NTA agarose column by reducing the concentration of urea. The refolding was confirmed by circular dichroism (CD) and ${ }^{1} \mathrm{H}$ NMR. By thermal unfolding experiments we show that a strand-strand cystine bridge has significant effect on the stability of the FLRT FnIII fold. We further show by Surface Plasmon Resonance that all 3 FnIII domains bind to FGFR1, and roughly estimate a $K_{d}$ for each domain, all $K_{d} s$ being in the $\mu \mathrm{M}$ range. 
1 Expression, refolding and spectroscopic characterization of Fibronectin type III 2 (FnIII)-homology domains derived from human Fibronectin Leucine Rich 3 Transmembrane Protein (FLRT)-1, -2, and -3

4

5

26

27

28

29

30

31

32

33

Lila Yang ${ }^{1 \S}$, Maria Hansen Falkesgaard $2 \S$, Peter Waaben Thulstrup ${ }^{1}$, Peter Schledermann Walmod $^{2}$, Leila Lo Leggio ${ }^{1}$ and Kim Krighaar Rasmussen ${ }^{1,2}$

${ }^{1}$ Biological Chemistry, Department of Chemistry, University of Copenhagen, Copenhagen, Denmark

${ }^{2}$ Laboratory of Neural Plasticity, Department of Neuroscience and Pharmacology, Faculty of Health and Medical Sciences, University of Copenhagen, Copenhagen, Denmark

$\S$ These authors have contributed equally

Corresponding author

Kim Krighaar Rasmussen ${ }^{1}$

Universitetsparken 5, Copenhagen, Denmark, 2100, Denmark

KKR@chem.ku.dk

Leila Lo Leggio ${ }^{1}$

Universitetsparken 5, Copenhagen, Denmark, 2100, Denmark

Leila@chem.ku.dk 
37 Abstract

38 The fibronectin leucine rich transmembrane (FLRT) protein family consists in 39 humans of 3 proteins, FLRT1, -2, and -3. The FLRT proteins contain 2 extracellular domains 40 separated by an unstructured linker. The most membrane distal part is a leucine rich repeat 41 (LRR) domain responsible for both cis- and trans-interactions, whereas the membrane 42 proximal part is a fibronectin type III (FnIII) domain responsible for a cis-interaction with 43 members of the fibroblast growth factor receptor 1 (FGFR1) family, which results in FGFR 44 tyrosine kinase activation.

45 Whereas the structures of FLRT LRR domains from various species have been 46 determined, the expression and purification of recombinant FLRT FnIII domains, important 47 steps for further structural and functional characterizations of the proteins, have not yet been 48 described. Here we present a protocol for expressing recombinant FLRT-FnIII domains in 49 inclusion bodies in Escherichia coli. His-tags permitted affinity purification of the domains, 50 which subsequently were refolded on a Ni-NTA agarose column by reducing the 51 concentration of urea. The refolding was confirmed by circular dichroism (CD) and ${ }^{1} \mathrm{H}-$ 52 NMR. By thermal unfolding experiments we show that a strand-strand cystine bridge has 53 significant effect on the stability of the FLRT FnIII fold. We further show by Surface 54 Plasmon Resonance that all 3 FnIII domains bind to FGFR1, and roughly estimate a $K_{\mathrm{d}}$ for 55 each domain, all $K_{\mathrm{d}} \mathrm{s}$ being in the $\mu \mathrm{M}$ range. 
68

69

70

71

72

73

74

75

76

77

78

79

80

81

82

83

84

85

86

87

88

89

90

91

92

93

94

95

96

97

98

\section{Introduction}

The fibronectin leucine rich transmembrane (FLRT) protein family was first described in 1999. In mammals it includes 3 proteins, FLRT1, -2, and -3 (Lacy et al., 1999) each comprised of an extracellular, membrane-distal leucine-rich repeat (LRR) domain (Seiradake et al., 2014) followed by a fibronectin type III (FnIII) domain, a transmembrane region and a cytoplasmic tail without obvious sequence motifs (Karaulanov, Böttcher \& Niehrs, 2006).

A number of extracellular binding partners to FLRT proteins have been reported, including Myelin-associated glycoprotein, Brother of $\mathrm{CDO}$, netrin receptors, and the synaptic, G-protein-coupled receptors, latrophilins (reviewed by Winther and Walmod 2014). Another group of proteins that interacts with FLRTs is the Fibroblast Growth Factor Receptor (FGFR) family. FGFRs constitute a family of trans-membrane receptors belonging to the receptor tyrosine kinases superfamily. They consist of an extracellular domain comprising 3 immunoglobulin (Ig) domains, a transmembrane domain (TMD), and an intracellular tail including a split tyrosine kinase domain. Ig domain 2 and 3 and the linker between them are known to be involved in binding to the classical FGFR ligands, FGFs, whereas Ig domain 1 has an autoinhibitory function on ligand binding (Gong, 2014). Following receptor dimerization, the intracellular kinase domains are trans-activated. The activated receptors in turn initiate downstream signaling through various pathways to generate diverse cellular responses, including cell growth, differentiation, migration and cell survival (Slack et al., 1996; Martin, 1998; Friesel \& Maciag, 1999; Groth \& Lardelli, 2002). Consequently, FGFR are implicated in a number of diseases including cancer, schizophrenia and Parkinson's disease (Jin et al., 2005; Krejci et al., 2009; Turner \& Grose, 2010; Haugsten et al., 2010; van Scheltinga, Bakker \& Kahn, 2010). In addition to their interaction with FGFs, the extracellular domains of FGFRs can bind several proteins located in the plasma membrane, including a number of cell adhesion molecules among which FLRTs.

The FnIII domain of Xenopus FLRT3 (XFLRT3) is reported to bind directly to FGFR1 (Böttcher et al., 2004), and a later study has demonstrated that all 3 mouse FLRTs act as regulators of FGFR-mediated signaling through direct interaction with FGFR1 independently of prior FGFR activation (Haines et al., 2006). Surprisingly, a more recent study implicates both the LRR and the cytoplasmic tail of mouse FLRT2 in a direct 
99 interaction with FGFR2 (Wei et al., 2011).

100 FLRT-FGFR-mediated signaling is important e.g. for craniofacial development (Wei

101 et al., 2011) and the folding of the cerebral cortex (del Toro et al., 2017). The 3 mammalian

102 FLRTs have different but occasionally overlapping expression patterns. Since they all can

103 bind e.g. FGFR1 any specificities in the signaling mediated by FLRT-FGFR interactions

104 would therefore require differences e.g. in the affinities between FLRTs and FGFRs. FLRT-

105 FGFR interactions, and in particular the interactions between FGFRs and FLRT FnIII 106 domains, need therefore to be studied in more detail.

107 The LRR regions of FLRT proteins have been produced recombinantly and 108 crystallized in complex with netrin receptors (Seiradake et al., 2014) and latrophilins 109 (Jackson et al., 2015). In contrast, the expression and purification of FLRT FnIII domains in 110 vitro have been so far not been reported and the detailed interaction between FGFR and 111 FLRTs remains to be elucidated.

112 Here we present a procedure for the expression and purification of FLRT FnIII 113 domains. One aim of the study was to eventually produce deuterated proteins suitable for 114 small angle neutron scattering, and hence Escherichia coli was chosen as an expression 115 system.

116

117

Materials and Methods

118 Construction and expression of FLRT-FnIII domains

119 Genes coding for human FLRT1, -2, and -3 were purchased from Source BioScience GmbH 120 (Germany). Using polymerase chain reaction (PCR) with oligonucleotides primer (FLRT1121 FnIII forward: 5'-GATGGCGCCAAGACCCTGGCC-3', 122 GgtaggGCCAtAgCtGTCGgC-3', FLRT2 FnIII forward: 5'123 сстAтtтCtgAACGgAtCCAGC-3', reverse: 5'-CAgAtAgGAgGCATGgGtgGt-3', FLRT3124 FnIII: 5'-AGTCCCTCAAGAAAAACAATTAC-3', 5'125 CAttcGaAgGgGtgCAgttTCAgt-3') cDNA for all three FLRT-FnIII domains was 126 amplified and cloned into pET-DEST42 Gateway (Invitrogen) destination vector containing 127 ampicillin resistance.

128

129 Protein expression 
130 All the constructs were transformed into E. coli BL21 (DE3) (Novagen) or Rosetta (DE3)

131 (Novagen) and selected with appropriated antibiotics. $10 \mathrm{ml}$ starter culture of Luria Bertani

132 (LB) broth containing $100 \mu \mathrm{g} / \mathrm{ml}$ ampicillin or $50 \mu \mathrm{g} / \mathrm{ml}$ kanamycin was inoculated with a

133 single colony, and grown at $37^{\circ} \mathrm{C}$ overnight with $250 \mathrm{rpm}$ shaking.

134 Afterwards, 1 L LB containing $100 \mu \mathrm{g} / \mathrm{ml}$ ampicillin or $50 \mu \mathrm{g} / \mathrm{ml}$ kanamycin was 135 inoculated with the overnight starter culture. The culture grew at $37{ }^{\circ} \mathrm{C}$ with $250 \mathrm{rpm}$ shaking 136 until an $\mathrm{OD}_{600}$ of 0.6 was reached. Overexpression was induced by adding isopropyl $\beta$-D-1137 thiogalactopyranoside (IPTG) to a final concentration of $1 \mathrm{mM}$, and the cultures were 138 allowed to overexpress for 20 hours at 15 or $25{ }^{\circ} \mathrm{C}$. The cells were harvested by 139 centrifugation at $6,000 \mathrm{x}$ g for $10 \mathrm{~min}$. Cell pellets were frozen for minimum $15 \mathrm{~min}$. at -20 $140{ }^{\circ} \mathrm{C}$. Thawed cell pellets were resuspended in denaturing buffer (8M urea, phosphate buffered

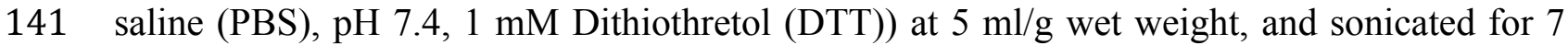
142 min. with $10 \mathrm{sec}$. pulse and $10 \mathrm{sec}$. pause with an amplitude set to $50 \%$. Following 143 sonication the cell paste was stirred for $60 \mathrm{~min}$. at room temperature to solubilize inclusion 144 bodies. The lysate containing soluble inclusion bodies was centrifuged at 20,000 x g for 30 $145 \mathrm{~min}$. at $+4{ }^{\circ} \mathrm{C}$ to pellet insoluble material. The supernatant was collected and filtered with $1460.45 \mu \mathrm{m}$ sterile filter before purification. $20 \%$ SDS-PAGE was used to follow purification 147 and verify protein purity with typical sample loading of $0.5-1 \mathrm{mg} / \mathrm{ml}$ protein. SDS-PAGE 148 gels were stained overnight with InstantBlue ${ }^{\mathrm{TM}}$ (Expedeon).

149

150 Purification and refolding of FLRT Fn III domains

151 FnIII domains from filtrates were immobilized on a $1 \mathrm{ml} \mathrm{HP}$ His Trap Ni-NTA column (1 for 152 each protein) (GE Healthcare) coupled to an Äkta purify system. The column was 153 equilibrated with 10-15 column volumes of denaturing buffer (8 M urea, PBS, $1 \mathrm{mM}$ DTT, $154 \mathrm{pH} \mathrm{7.4)} \mathrm{with} \mathrm{flow} \mathrm{rate} 1 \mathrm{ml} / \mathrm{min}$. Following equilibration the filtrate was loaded onto the 155 column, and washed with wash buffer (8 M urea, PBS, $1 \mathrm{mM}$ DTT, $20 \mathrm{mM}$ imidazole, pH 156 7.4) for at least 30 column volumes until the $A_{280}$ - and $A_{260} \mathrm{~nm}$ were stabilized. 157 Subsequently, the washed proteins were refolded on the column by applying PBS ( $\mathrm{pH} 7.4)$, 158 thus generating a decreasing gradient of urea and DTT (from $8 \mathrm{M}$ urea and $1 \mathrm{mM}$ DTT to 0 $159 \mathrm{M}$ in $30 \mathrm{~min}$.). A second wash step was included with wash buffer (5 x PBS and $20 \mathrm{mM}$ 160 imidazole $\mathrm{pH}$ 7.4). Before eluting the protein, PBS was run through the column to decrease 
161 the salt concentration. The protein was eluted with elution buffer (PBS pH 7.4 containing 500

$162 \mathrm{mM}$ imidazole) for 5 column volumes. The purification was monitored using ultraviolet 163 absorption at $280 \mathrm{~nm}$ and $260 \mathrm{~nm}$. The refolded proteins were further purified by size164 exclusion chromatography (SEC) using a $320 \mathrm{ml}$ or $120 \mathrm{ml}$ Superdex 75 16/60 GL column 165 equilibrated with regular PBS. The collection was monitored at $\mathrm{A}_{280}$ - and $\mathrm{A}_{260} \mathrm{~nm}$. All 166 refolding and purification procedures were carried out at $4{ }^{\circ} \mathrm{C}$ and the purified proteins were 167 stored at $-80^{\circ} \mathrm{C}$ until use.

168

169 Characterization of FLRT-FnIII domains

170 Matrix-assisted laser desorption/ionization time-of-flight (MALDI-TOF)

171 The masses of the FLRT domains were determined by MALDI-TOF-MS. $1 \mu 1$ sample and 1 $172 \mu \mathrm{L}$ a-Cyano-4-hydroxycinnamic acid (HCCA) matrix were mixed and dispensed onto a 173 MALDI target and allowed to dry out at room temperature. Protein masses were acquired in 174 linear mid-mass positive mode, averaging 2,000 laser shots per MALDI-TOF spectrum. 175 Calibration mixtures (Sigma-Aldrich) were used to calibrate the spectrum to a mass tolerance 176 within $2 \mathrm{Da}$. The mass range was 5,000-14,000 Da.

177

\section{Circular dichroism spectroscopy}

179 The Far-UV CD measurements were performed using a Jasco J-815 spectropolarimeter at 20 $180{ }^{\circ} \mathrm{C}$ in a $1 \mathrm{~mm}$ path length quartz cuvette from Hellma over the wavelength range 190-260 $181 \mathrm{~nm}$. A resolution of $1 \mathrm{~nm}$ and a scan speed of $20 \mathrm{~nm} / \mathrm{min}$ were employed. At least 3 182 consecutive scans were obtained, the resulting data was smoothed and the contribution of the 183 buffer blank was subtracted. The monitored scans were averaged. Spectra were analyzed for 184 secondary structural distribution using the Beta Structure Selection, BeStSel, server 185 (Micsonai et al., 2015). Variable temperature measurements were carried out in the absence 186 or presence DTT within the temperature ranges of $20-90$ and $90-20{ }^{\circ} \mathrm{C}$, at fixed wavelengths 187 of 213 and $228.5 \mathrm{~nm}$. Monitoring of $\mathrm{CD}$ at both wavelengths gave comparable results, but as 188 the $\mathrm{CD}$ signal at $228.5 \mathrm{~nm}$ had a higher magnitude these data are presented. The temperature189 dependent CD signal was normalized to vary between 1 and 0 for the initial and final data in 190 the series for each protein sample, representing a fully folded and fully unfolded state. The 191 (linear) temperature dependency of folded or unfolded protein was not taken into account. 
192

$1931 D-N M R$

194 For 1D-NMR measurements, $50 \mu \mathrm{D}_{2} \mathrm{O}$ (Invitrogen) and $500 \mu \mathrm{l}$ of the concentrated sample 195 of protein were transferred to an NMR tube. Following the preparation of NMR samples a 196 1D-spectrum was recorded at $298 \mathrm{~K}$ on a Varian $750 \mathrm{MHz}$ spectrometer using a cold probe.

198 Template-based protein structure prediction

199 Primary sequences of all 3 FnIII domains (FLRT1; $200{ }^{405}$ GDGAKTLA IHVKALTADS IRITWKATLPASS FRLSWLRLGHSPAVGS ITETLVQGDKTEYLL

201 TALEPKSTYIICMVTMETSNAYVADETPVCAKAETADSYGPT ${ }^{508,}$ FLRT2;

$202{ }^{420}$ GPISERIQLS IHFVNDTS IQVSWLSLFTVMAYKLTWVKMGHSLVGG IVQERIVSGEKQHLSL 203 VNLEPRSTYRICLVPLDAFNYRAVEDTICSEATTHASYL519 and FLRT3; $204{ }^{404}$ GSPSRKTITITVKSVTSDTIHISWKLALPMTALRLSWLKLGHSPAFGS ITETIVTGERSEYL 205 VTALEPDSPYKVCMVPMETSNLYLFDETPVCIETETAPLRM ${ }^{50}$ ) were input to the I-TASSER 206 server (Yang et al., 2014) for template-based modeling.

207

208

Interactions studies with Fibroblast Growth Factor Receptor1 (FGFR1)

209 Binding studies were performed using a BIAcore 2000 instrument (BIAcore) at $25^{\circ} \mathrm{C}$ using 10 210 mM PBS ( $\mathrm{pH}$ 7.4) as running buffer. A CM4 sensor chip was immobilized with approximately 2111300 RU of FGFR1 $\beta$ (IIIc)/Fc chimera (R\&D Systems) and 750 RU recombinant human $\operatorname{IgG}_{1}$ 212 Fc, Cat. No. 110-HG (carrier-free, R\&D Systems). The FGFR1 and IgG $_{1}$ proteins were 213 immobilized by an amine coupling kit (BIAcore) with flowrate $5 \mu 1 / \mathrm{min}$. FLRT1, -2 and -3 FnIII 214 domains in PBS ( $\mathrm{pH} 7.4$ ) were injected at $20-30 \mu 1 / \mathrm{min}$. The binding curves were analyzed by 215 BIAevaluation version 4.1 software. A steady state affinity analysis was used to estimate $K_{d}$.

216

217 Result and Discussion

218 Expression of FLRT Fn IIIs

219 Purified pET-DEST42 destination vectors containing each relevant non-codon optimized 220 FLRT FnIII gene were transformed into E.coli strain BL21 (DE3) or the rare codon harboring 221 Rosetta (DE3), which were then used as expression hosts of recombinant FnIII domains for 222 all 3 FLRT domains. As standard conditions for protein expression proteins, the strains 
223 harboring the plasmids were cultured at $37{ }^{\circ} \mathrm{C}$ until an $\mathrm{OD}_{600}$ of 0.6 was reached. At this

224 density the cultures were induced with IPTG, and temperature was adjusted for expression.

225 However, no obvious overexpression could be visualized for any of the constructs. Although

226 several expression parameters (different temperatures, E. coli bacterial strains, and $\mathrm{OD}_{600}$

227 values before IPTG induction) were tested, the non-codon optimized genes cloned in to the

228 pET-DEST42 vector gave no (FLRT2 FnIII) or little (FLRT1 and FRLT3 FnIII) protein

229 expression. Furthermore, the various changes in expression conditions did not result in 230 soluble protein, and the FnIII domains were always expressed as inclusion bodies.

231 Since attempts to express the recombinant proteins from non-codon optimized

232 constructs had limited success, codon optimized genes were designed and purchased from

233 GenScript (USA). The genes were synthesized and cloned into the pET-30-a(+), a vector that

234 like pET-DEST42 contains the bacteriophage T7 polymerase promoter (Studier \& Moffatt,

235 1986). However, pET-30-a(+) and pET-DEST42 contain different antibiotic resistance

236 (kanamycin and ampicillin, respectively).

237 In contrast to the former expression experiments with the non-codon optimized 238 constructs, the codon optimized constructs were expressed with higher yields. The proteins 239 were still expressed as inclusion bodies as seen from small-scale test expressions. Thus, 240 refolding was still required. The final protocol used for expression of inclusion bodies was to 241 grow the BL21 (DE3) culture at $37^{\circ} \mathrm{C}$ to an $\mathrm{OD}_{600}=0.6$ where $1 \mathrm{mM}$ IPTG was added to 242 induce recombinant protein expression. The culture was allowed to express overnight.

243

244 Purification of FLRT Fn III domains

245 From the non-optimized gene constructs it was only possible to express the FnIII domains 246 from FLRT1 and FLRT3, but despite very low yields, a purification procedure could be 247 established. Since the recombinant proteins were localized in inclusion bodies, the bacteria 248 pellets were solubilized in denaturation buffer containing $8 \mathrm{M}$ urea followed by 249 immobilization and refolding on-column using a Ni NTA column, and by decreasing the 250 concentration of urea to $0 \mathrm{M}$. After washing, proteins were eluted and further purified using 251 SEC (see Fig. 1). From a 11 culture 1-2 mg protein was finally obtained for further 252 characterization. The purities of the recombinant proteins were validated by SDS-PAGE, 253 which demonstrated that the proteins were $\sim 99 \%$ pure protein (see Fig. 2). Initially, refolding 
254 was performed without DTT. However, all 3 FLRT FnIII domains contain 2 cysteines. Thus,

255 DTT was included in the denaturation buffer. The addition of DTT had a significant effect on 256 the monomer/dimer ratio as determined by SEC, (see Fig.1, (green curves)).

257

258 Characterization of FLRT-FnIIIs

259 Mass spectrometry

260 For all 3 FnIII domains, MALDI-TOF-MS analysis was performed to ensure correct 261 recombinant protein expression as well as pure sample (see Table 1 and Supplementary Table 2621 and Fig. 3). Methionine (Met) has previously been shown to be artificially oxidized due to 263 sample handling both for MALDI-TOF and ESI measurements (Chen \& Cook, 2007; 264 Thirumangalathu et al., 2007), thus while analyzing theoretical and experimental masses, 265 oxidation of one or more Met residues was allowed.

266 For FLRT3-FnIII MALDI-TOF we observed 1 peak (12232.2 Da) corresponding to

267 the loss of the N-terminal Met. It has previously been shown that, when a small amino acid 268 like glycine is following the N-terminal Met, Met is likely to be cleaved off (Frottin et al., 269 2006). For FLRT2-FnIII 3 peaks were observed, 1 at $11746.3 \mathrm{Da}$ corresponding to the N270 terminal degraded by 6 residues (1-MGPISE-6) see Table S1, and the other 2 at $11789.5 \mathrm{Da}$ 271 and 11827.7 Da most likely corresponding to either carbamylation (+43 Da) from the urea or 272 acetylation $(+42 \mathrm{Da})$, and the same post-translational variant with a bound potassium ion $273(+38 \mathrm{Da})$ see Fig 3. In the case of FLRT1-FnIII 5 peaks were observed at $11122.8 \mathrm{Da}$, 274 11254.2 Da, 11947.6 Da, 12077.3 Da and 12106.0 Da (see Fig. 3). Here we observe 2 N275 terminally degraded variants (11122.8 Da and 11254.2 Da), an N-terminal Methionine 276 cleaved variant (11947.6 Da), a full length FLRT1-FnIII (12077.3 Da) see Table S1, and a 277 mass of $+28 \mathrm{Da}$ from the full length (not identified here). In conclusion these results 278 demonstrate that the recombinant proteins are correctly expressed, but that they are prone to 279 N-terminal degradation. Notably, the proteins were not stored with protease inhibitors. 280 Addition of protein inhibitors would possibly reduce the degree of the observed N-terminal 281 degradation.

282

283 Structural characterization by Circular Dichroism and NMR Spectroscopy

284 Far-ultraviolet CD spectra measurements were performed on all 3 FnIII domains to confirm 
285 that the refolding had been successful (Fig. 4). The spectra of the FLRT1- and FLRT3 FnIII

286 domains were almost identical with deviations only at wavelengths below $208 \mathrm{~nm}$. The 287 spectra are typical of a classical $\beta$-sheet signal with a right twist of the sheet influencing the 288 spectrum from a (positive) maximum at $\sim 230 \mathrm{~nm}$ to a (negative) minimum around $215 \mathrm{~nm}$ 289 (Micsonai et al., 2015). The maximum $\sim 230 \mathrm{~nm}$ in the profile may have contributions from 290 the aromatic side chains as discussed in earlier studies of other FnIII domains (Stevens et al., 291 1987; Brumfeld \& Werber, 1993). The spectra thus clearly show that the two domains are 292 folded. Furthermore a 1D proton NMR spectrum, ${ }^{1} \mathrm{H}-\mathrm{NMR}$, of FLRT1-FnIII (see Fig. S1) 293 resulted in broad dispersion of peaks with two peaks at $10.2 \mathrm{ppm}$ and $10.0 \mathrm{ppm}$ arising from 294 the 2 tryptophan $\mathrm{N} \varepsilon$ protons. The 2 different chemical shifts clearly show that these 295 tryptophans have different local environment and also support the interpretation that the 296 produced FLRT1-FnIII domain is folded. The CD spectrum of FLRT2-FnIII deviates from 297 the spectra of the other 2 proteins. However, it also has a maximum at $\sim 230 \mathrm{~nm}$ indicating 298 that the domain is folded. CD signals from antiparallel $\beta$-sheet are relatively weak compared 299 to an $\alpha$-helical signal. The CD profile of FLRT2 FnIII still has an antiparallel $\beta$-sheet signal, 300 but in contrast to the FLRT1 and FLRT3 FnIII domains, the FLRT2 FnIII domain is 301 estimated to contain a larger fraction of the antiparallel $\beta$-sheet (47.6\%). Of this fraction 30.2 $302 \%$ is estimated to be anti3 (right-twisted) $\beta$-sheet, which is $6-7 \%$ more than for the FLRT1 303 and FLRT3 FnIII domains (see Table 2). This could explain the signal differences between 304 the CD profiles from the FnIII domains. Due to the differences in the CD spectra it cannot be 305 excluded that a larger part of FLRT2 is folded differently compared to FLRT1 and FLRT3, or 306 that the protein is only partially folded.

307

308

\section{Stability of the FnIII domains}

309 Due to the differences in CD spectra it could not be excluded that FLRT2 FnIII might only be 310 partially folded. In order to investigate this possibility, thermal unfolding of all three domains was performed by variable temperature measurement at a fixed wavelength $(228.5 \mathrm{~nm})$ where a strong CD signal was observed (see Fig5; see Fig. 4 for the full CD spectra). Both FLRT1

313 FnIII and FLRT3 FnIII unfolded at $\sim 68-69{ }^{\circ} \mathrm{C}$. In contrast FLRT2 FnIII unfolded at $\sim 42{ }^{\circ} \mathrm{C}$. 314 To explain this significant difference we hypothesized that a Cys-Cys bridge from strand $\mathrm{F}$ to 315 strand G is stabilizing the FnIII domain of FLRT1 and FLRT3, but not FLRT2. To test this 
316 hypothesis, identical variable temperature CD measurements were recorded with the only 317 difference that $1 \mathrm{mM}$ DTT had been added to the samples. The addition of DTT had a 318 significant impact on the unfolding of FLRT1 FnIII and FLRT3 FnIII. In the presence of 319 DTT the FLRT1 and FLRT3 FnIII domains unfolded at $\sim 48$ and $38{ }^{\circ} \mathrm{C}$, respectively. They 320 were destabilized to a degree where a plateau at low temperature $\left(20^{\circ} \mathrm{C}\right)$ was not established, 321 making a proper $\mathrm{T}_{\mathrm{m}}$ value difficult to assess. In the case of FLRT1 FnIII domain the 322 signal/noise was relatively low, but it is clear that in the presence of DTT the domain was 323 less stable. In contrast, the addition of DTT did not affect the unfolding temperature of 324 FLRT2 FnIII domain (see Fig. 5, C). The domain still unfolds with $\mathrm{T}_{\mathrm{m}} \sim 42{ }^{\circ} \mathrm{C}$, indicating the 325 absence of disulphide formation although two cysteines are present on $\mathrm{F}$ and $\mathrm{G}$ strands as for 326 the other two domains (see Fig. 5, C). A sequence alignment of the 3 FnIII domains shows 327 that the FLRT2 FnIII domain lacks a residue between the Cys residues, when compared to 328 FnIII domains of FLRT1 and FLRT3 (see Fig. S2). In the case of FLRT2 it could be a 329 distance restriction that prevents the formation of a cysteine bridge. Another, but less likely 330 situation would be, that the disulphide bridge is formed, but does not contribute further to the 331 stability of the FLRT2 FnIII domain. The order of stability of FnIII domains in the presence 332 of DTT is FLRT3<FLRT2<FLRT1. The differences, especially between FLRT1 and FLRT3, 333 which are quite significant, could be due to the differences between the hydrophobic cores of 334 the domains. From the sequence alignment of the domains it can be hypothesized that the 335 extra aromatic residues in the FLRT1 FnIII domain stabilizes the core more, when compared 336 to the FLRT3 FnIII domain. A final explanation for the results obtained is that the FLRT2 337 FnIII domain is incorrectly folded, so that the native Cys-Cys bridge cannot be formed, 338 giving a rationale for the differences in CD-spectra compared to the two other FnIII domains. 339 From mass spectrometry it was possible to observe N-terminal terminal cleavage of FLRT2 340 FnIII, which might affect the folding, or be a consequence of partial unfolding. In each case, 341 with or without DTT, the measurement was carried out from 90 to $20^{\circ} \mathrm{C}$ to determine the 342 folding reversibility. However, none of the domains exhibited any reversibility and thus 343 remained unfolded after the first thermal unfolding.

344

345 Template-based protein structure prediction of FLRTs-FnIII domains

346 With few exceptions (Huber et al., 1994), FnIII domains of known structures do not contain 
347 Cys-Cys bridges, while the FLRT domains investigated here do. The Cys residues are at 348 position 75 and 93 in the sequences of FLRT1 and FLRT3 FnIII, and position 75 and 92 in 349 FLRT2 FnIII. Since the stability investigations indicate a possible involvement of disulphide 350 bridges in the stability, we wanted to investigate if the spatial arrangement allows for Cys351 Cys bridging by performing template-based protein structure prediction using I-TASSER. A 352 detailed description of I-TASSER is given elsewhere (Zhang, 2007; Wu, Skolnick \& Zhang, 353 2007; Yang et al., 2014). Briefly, I-TASSER predicts protein structures from primary 354 sequences, first by identifying structural templates from the PDB library with LOMETS, next 355 by performing an iterative Monte Carlo simulation and model refinement to construct the 356 topology of the protein. Up to 5 models are constructed and validated by confidence score 357 (C-score). C-scores should be in the range between -5 and 2, with more positive values being 358 the better and C-scores over -1.5 indicating usually a correct fold. The best models all had C359 scores better than -0.5 see Table S2. For the FLRT2 FnIII domain we obtained 4 models, 360 which is a good sign of convergence, and thus reliability of the model. I-TASSER also 361 returns a TM score, which is a sequence length-independent metric for measuring structure 362 similarity in the same SCOP/CATH fold family. The TM values were in the range between 0 363 and 1 (a TM-score $>0.5$ indicating a model with correct topology and a TM-score $<0.17$ 364 indicating random similarity). For all 3 FnIII domains the models scored TM values $>0.66$ 365 (see Table S2). The best I-TASSER model is used in a search against structure in the PDB 366 library to identify structural homologues. This search is different compared to the former, 367 which is a sequence-based threading search while the latter is a structural search.

Several structures of FnIII domains have been solved within the last decades, and 369 despite high tertiary structure similarities they tend to share low sequence identity. This is 370 also evident from the I-TASSER structure prediction where sequence identities with the used 371 templates range from 12.6 to $23.2 \%$. All top solutions had sequence coverage from 91.3 to $37294.1 \%$. The tertiary structure of the FnIII domains consist of a $\beta$-sheet built up by B, C, E 373 and $F \quad \beta$-strands, and a $\beta$-sheet composed of $A, C^{\prime}$ and $G \beta$-strands. A hydrophobic core 374 stabilizes the domain. The topology of the FnIII domain has previously been shown to have 375 conserved Trp residues as well as a hydrophobic pattern building up the hydrophobic core. 376 When inspecting the sequences of the FLRTs FnIII domains it can be seen the also contain 377 this hydrophobic pattern (see Figure S3). Taken together, the modelling of the FLRT FnIIIs 
378 seems reliable, within the limits imposed by low sequence identity with the used templates.

379 All models modelled by I-TASSER placed the 2 Cys residues in close vicinity, even though

380 the templates used for modeling did not contain cysteines. This suggests that a disulphide

381 bridge could be formed between the F and G strand (see Fig 5), explaining the effect of DTT

382 in the stability of the FLRT1 and FLRT3 FnIII domains. For the FLRT2 FnIII domain the

383 situation is not as clear. The thermal unfolding showed no effect of DTT, and this could be

384 due to partial unfolding or misfolding, but a disulphide bond may be formed in vivo.

Interactions between with FGFRI and FLRT FnIII domains

387 Since the Xenopus FLRT3 FnIII domain has been shown in vivo by truncation experiments to 388 interact with FGFR1 (Böttcher et al., 2004), we tried to see if interaction between human 389 FLRT FnIII domains and FGFR1 could be demonstrated in vitro. Surface plasmon resonance 390 (SPR) analysis with FGFR1 $\beta$ immobilized on the chip, and FLRT FnIII domains in solution 391 demonstrated the expected binding between FGFR1 $\beta$ and FLRT FnIIIs domains (see Fig. 392 S4). On the same chip an Ig domain had been immobilized as negative control to detect 393 unspecific binding. No unspecific binding was observed for any of the tested FnIII domains 394 (see Fig. S4). By steady state fitting we roughly estimated the $\mathrm{K}_{\mathrm{d}}$ 's for the interaction 395 between FGFR $1 \beta$ and the FnIII domains. The $\mathrm{K}_{\mathrm{d}} \mathrm{s}$ are all in the $\mu \mathrm{M}$ range (see Fig. S4), 396 which corresponds to $\mathrm{K}_{\mathrm{d}}$ values obtained for other FGFR-FnIII domains interactions 397 (Christensen et al., 2006). The $\mathrm{K}_{\mathrm{d}} \mathrm{s}$ should only be taken as indicative as the measurements 398 were only performed twice. Interestingly, although the FLRT2 FnIII domain shows lower 399 affinity than the other two, the $\mathrm{K}_{\mathrm{d}}$ is in a similar range suggesting that the domain is at least 400 partly correctly folded.

401

402 Conclusion

403 Here we present a protocol for expression and on-column refolding of FLRT FnIII domains. 404 It can be concluded that codon optimization had significant effects on the expression levels, 405 when compared to non-codon optimized constructs. Moreover, it can be concluded that on406 column refolding of FLRTs-FnIII domains can be performed successfully as seen from CD 407 experiments and NMR studies, but also that protease inhibitors should be included in future 408 studies, since the domains are prone to cleavage. Also, we concluded from a combination of 
409 modelling and stability studies, that a Cys-Cys bridge between the F and G strands might be 410 necessary to stabilize the FnIII domains. Finally, we show that all 3 recombinant human 411 FLRT FnIII domains in vitro bind FGFR1b with $K_{d}$ values in the $\mu \mathrm{M}$ range. Binding of 412 FLRT proteins to FGFRs have been reported to include both extracellular and intracellular 413 interactions (Wei et al., 2011), but our data demonstrate that FLRT FnIII domains alone are 414 sufficient to mediate FLRT-FGFR interaction in vitro. We speculate that differences in the 415 primary, secondary or tertiary structures of FLRT FnIII domains might contribute to different 416 binding affinities between different members of FLRT and FGFR families, hence ensuring 417 variability in the biological responses to FLRT-FGFR interactions.

418

419 Acknowledgements

420 We will like to thanks Christian Tortzen for assistance using the NMR spectrometer, and 421 laboratory technicians Annette Andersen and Uriwan Ngamrabiab Adamsen, for assistance 422 using mass spectrometry. Also we like to thanks Novo Nordisk contact Frederik Öberg for 423 discussions.

424

425

426

427

428

429

430

431

432

433

434

435

436

437

438

439

References 
440 Böttcher RT., Pollet N., Delius H., Niehrs C. 2004. The transmembrane protein XFLRT3 441 forms a complex with FGF receptors and promotes FGF signalling. Nature Cell 442 Biology 6:38-44. DOI: 10.1038/ncb1082.

443 Brumfeld V., Werber MM. 1993. Studies on Fibronectin and Its Domains. Archives of 444 Biochemistry and Biophysics 302:134-143. DOI: 10.1006/abbi.1993.1191.

445 Chen M., Cook KD. 2007. Oxidation Artifacts in the Electrospray Mass Spectrometry of A $\beta$ 446 Peptide. Analytical Chemistry 79:2031-2036. DOI: 10.1021/ac061743r.

447 Christensen C., Lauridsen JB., Berezin V., Bock E., Kiselyov VV. 2006. The neural cell 448 449 adhesion molecule binds to fibroblast growth factor receptor 2. FEBS letters 580:3386-3390. DOI: 10.1016/j.febslet.2006.05.008.

450 Friesel R., Maciag T. 1999. Fibroblast growth factor prototype release and fibroblast growth 451 factor receptor signaling. Thrombosis and Haemostasis 82:748-754.

452 Frottin F., Martinez A., Peynot P., Mitra S., Holz RC., Giglione C., Meinnel T. 2006. The 453 Proteomics of N-terminal Methionine Cleavage. Molecular \& Cellular Proteomics 454 5:2336-2349. DOI: 10.1074/mcp.M600225-MCP200.

455 Gong S-G. 2014. Isoforms of receptors of fibroblast growth factors. Journal of Cellular 456 Physiology 229:1887-1895. DOI: 10.1002/jcp.24649.

457 Groth C., Lardelli M. 2002. The structure and function of vertebrate fibroblast growth factor 458 receptor 1. The International Journal of Developmental Biology 46:393-400. of FLRT genes implies a functional role in the regulation of FGF signalling during mouse development. Developmental Biology 297:14-25. DOI: 10.1016/j.ydbio.2006.04.004. 
463 Haugsten EM., Wiedlocha A., Olsnes S., Wesche J. 2010. Roles of fibroblast growth factor 464 465 466 467 468 469 470

Huber AH., Wang YM., Bieber AJ., Bjorkman PJ. 1994. Crystal structure of tandem type III 10.1158/1541-7786.MCR-10-0168. receptors in carcinogenesis. Molecular cancer research: MCR 8:1439-1452. DOI: fibronectin domains from Drosophila neuroglian at 2.0 A. Neuron 12:717-731. Structural Basis of Latrophilin-FLRT Interaction. Structure 23:774-781. DOI: 10.1016/j.str.2015.01.013.

Jin K., LaFevre-Bernt M., Sun Y., Chen S., Gafni J., Crippen D., Logvinova A., Ross CA., Greenberg DA., Ellerby LM. 2005. FGF-2 promotes neurogenesis and neuroprotection and prolongs survival in a transgenic mouse model of Huntington's

476 Karaulanov EE., Böttcher RT., Niehrs C. 2006. A role for fibronectin-leucine-rich 477 478 disease. Proceedings of the National Academy of Sciences of the United States of America 102:18189-18194. DOI: 10.1073/pnas.0506375102. transmembrane cell-surface proteins in homotypic cell adhesion. EMBO reports 478 479 480 481 7:283-290. DOI: 10.1038/sj.embor.7400614.

Krejci P., Prochazkova J., Bryja V., Kozubik A., Wilcox WR. 2009. Molecular pathology of the fibroblast growth factor family. Human Mutation 30:1245-1255. DOI: 10.1002/humu.21067. and FLRT3: A Novel Family of Transmembrane Leucine-Rich Repeat Proteins. Genomics 62:417-426. DOI: 10.1006/geno.1999.6033. 
485 Martin GR. 1998. The roles of FGFs in the early development of vertebrate limbs. Genes \&

486

487

488

489

490

491

492

493

494

495

496

497

498

499

500

501

502

503

504

505

506
Development 12:1571-1586. DOI: 10.1101/gad.12.11.1571.

Micsonai A., Wien F., Kernya L., Lee Y-H., Goto Y., Réfrégiers M., Kardos J. 2015. Accurate secondary structure prediction and fold recognition for circular dichroism spectroscopy. Proceedings of the National Academy of Sciences of the United States of America 112:E3095-3103. DOI: 10.1073/pnas.1500851112.

van Scheltinga AFT., Bakker SC., Kahn RS. 2010. Fibroblast Growth Factors in Schizophrenia. Schizophrenia Bulletin 36:1157-1166. DOI: 10.1093/schbul/sbp033.

Seiradake E., del Toro D., Nagel D., Cop F., Härtl R., Ruff T., Seyit-Bremer G., Harlos K., Border EC., Acker-Palmer A., Jones EY., Klein R. 2014. FLRT Structure: Balancing Repulsion and Cell Adhesion in Cortical and Vascular Development. Neuron 84:370385. DOI: 10.1016/j.neuron.2014.10.008.

Slack JM., Isaacs HV., Song J., Durbin L., Pownall ME. 1996. The role of fibroblast growth factors in early Xenopus development. Biochemical Society Symposium 62:1-12.

Stevens ES., Morris ER., Charlton JA., Rees DA. 1987. Vacuum ultraviolet circular dichroism of fibronectin. Dominant tyrosine effects. Journal of Molecular Biology 197:743-745.

Studier FW., Moffatt BA. 1986. Use of bacteriophage T7 RNA polymerase to direct selective high-level expression of cloned genes. Journal of Molecular Biology 189:113-130.

Thirumangalathu R., Krishnan S., Bondarenko P., Speed-Ricci M., Randolph TW., Carpenter JF., Brems DN. 2007. Oxidation of Methionine Residues in Recombinant Human Interleukin-1 Receptor Antagonist: Implications of Conformational Stability on Protein Oxidation Kinetics ${ }^{\dagger}$. Biochemistry 46:6213-6224. DOI: 10.1021/bi700321g. 
507 del Toro D., Ruff T., Cederfjäll E., Villalba A., Seyit-Bremer G., Borrell V., Klein R. 2017.

508 Regulation of Cerebral Cortex Folding by Controlling Neuronal Migration via FLRT

509 Adhesion Molecules. Cell 169:621-635.e16. DOI: 10.1016/j.cell.2017.04.012.

510 Turner N., Grose R. 2010. Fibroblast growth factor signalling: from development to cancer.

$511 \quad$ Nature Reviews. Cancer 10:116-129. DOI: 10.1038/nrc2780.

512 Wei K., Xu Y., Tse H., Manolson MF., Gong S-G. 2011. Mouse FLRT2 Interacts with the

513 Extracellular and Intracellular Regions of FGFR2. Journal of Dental Research

514 90:1234-1239. DOI: 10.1177/0022034511415272.

515 Wu S., Skolnick J., Zhang Y. 2007. Ab initio modeling of small proteins by iterative TASSER

516 simulations. BMC biology 5:17. DOI: 10.1186/1741-7007-5-17.

517 Yang J., Yan R., Roy A., Xu D., Poisson J., Zhang Y. 2014. The I-TASSER Suite: protein

$518 \quad$ structure and function prediction. Nature Methods 12:7-8. DOI:

$519 \quad 10.1038 /$ nmeth.3213.

520 Zhang Y. 2007. Template-based modeling and free modeling by I-TASSER in CASP7.

$521 \quad$ Proteins 69 Suppl 8:108-117. DOI: 10.1002/prot.21702.

522

523

524

525

526

527

528

529

530

531 Table 1

532 


\begin{tabular}{|c|c|c|c|c|}
\hline & Peak (charge) & Peak (charge) & Peak (charge) & Peak (charge) \\
\hline \multicolumn{5}{|c|}{ MALDI-TOF } \\
\hline FLRT1 & 12077.3 & 11947.6 & 11254.2 & 11122.8 \\
\hline $\begin{array}{l}\text { Theoretical } \\
\text { masses }\end{array}$ & 12073.72 & 12073.72 & 12073.72 & 12073.72 \\
\hline $\begin{array}{l}\text { Deviation } \\
\text { from Theo. }\end{array}$ & 3.58 & 126.12 & 819.52 & 950.92 \\
\hline FLRT2 & 11827.7 & 11789.5 & 11746.3 & \\
\hline $\begin{array}{l}\text { Theoretical } \\
\text { masses }\end{array}$ & 12335.15 & 12335.15 & 12335.15 & \\
\hline $\begin{array}{l}\text { Deviation } \\
\text { from Theo. }\end{array}$ & 507.45 & 545.65 & 588.85 & \\
\hline FLRT3 & 12230.8 & & & \\
\hline $\begin{array}{l}\text { Theoretical } \\
\text { masses }\end{array}$ & 12356.27 & & & \\
\hline $\begin{array}{l}\text { Deviation } \\
\text { from Theo. }\end{array}$ & 125.47 & & & \\
\hline
\end{tabular}

533

534 Table 1. Masses determined from MALDI-TOF. Theoretical masses are calculated from

535 sequences given in material and methods including N-terminal Methionine and C-terminal His $6^{-}$ 536 tag.

537

538

539

540

541

542

543

544

545

546

547

548

549

550

Table 2 


\begin{tabular}{llllll}
\hline & Helix & Antiparallel & Parallel & Turn & Others \\
\hline FLRT1FnIII & 1.9 & 43.3 & 0.0 & 9.0 & 45.8 \\
FLRT2FnIII & 0.0 & 47.6 & 0.0 & 8.4 & 43.9 \\
FLRT3FnIII & 0.0 & 41.5 & 0.0 & 6.5 & 51.9 \\
\hline
\end{tabular}

552

553 Degree twisted

\begin{tabular}{llllll}
\hline & Helix1 & Helix2 & $\begin{array}{l}\text { Anti1 } \\
\text { (left- } \\
\text { twisted) }\end{array}$ & $\begin{array}{l}\text { Anti2 } \\
\text { (relaxed) }\end{array}$ & $\begin{array}{l}\text { Anti3 } \\
\text { (right- } \\
\text { twisted) }\end{array}$ \\
\hline FLRT1FnIII & 0.7 & 1.2 & 0.8 & 19.5 & 23.0 \\
FLRT2FnIII & 0.0 & 0.0 & 0.0 & 17.4 & 30.2 \\
FLRT3FnIII & 0.0 & 0.0 & 0.0 & 17.7 & 23.9 \\
\hline
\end{tabular}

554

555 Table 2. Estimated secondary structure content. The BESTSEL server (Micsonai et al., 2015) is 556 developed to characterize secondary structure content from CD spectra. It is especially 557 developed for analysis of antiparallel $\beta$-sheet folded proteins. Thus, it provides estimates of the 558 percentages of left- (Anti1) and right-twisted (Anti3) as well relaxed (Anti2) antiparallel $\beta$ 559 sheets. 
FIGURE 1

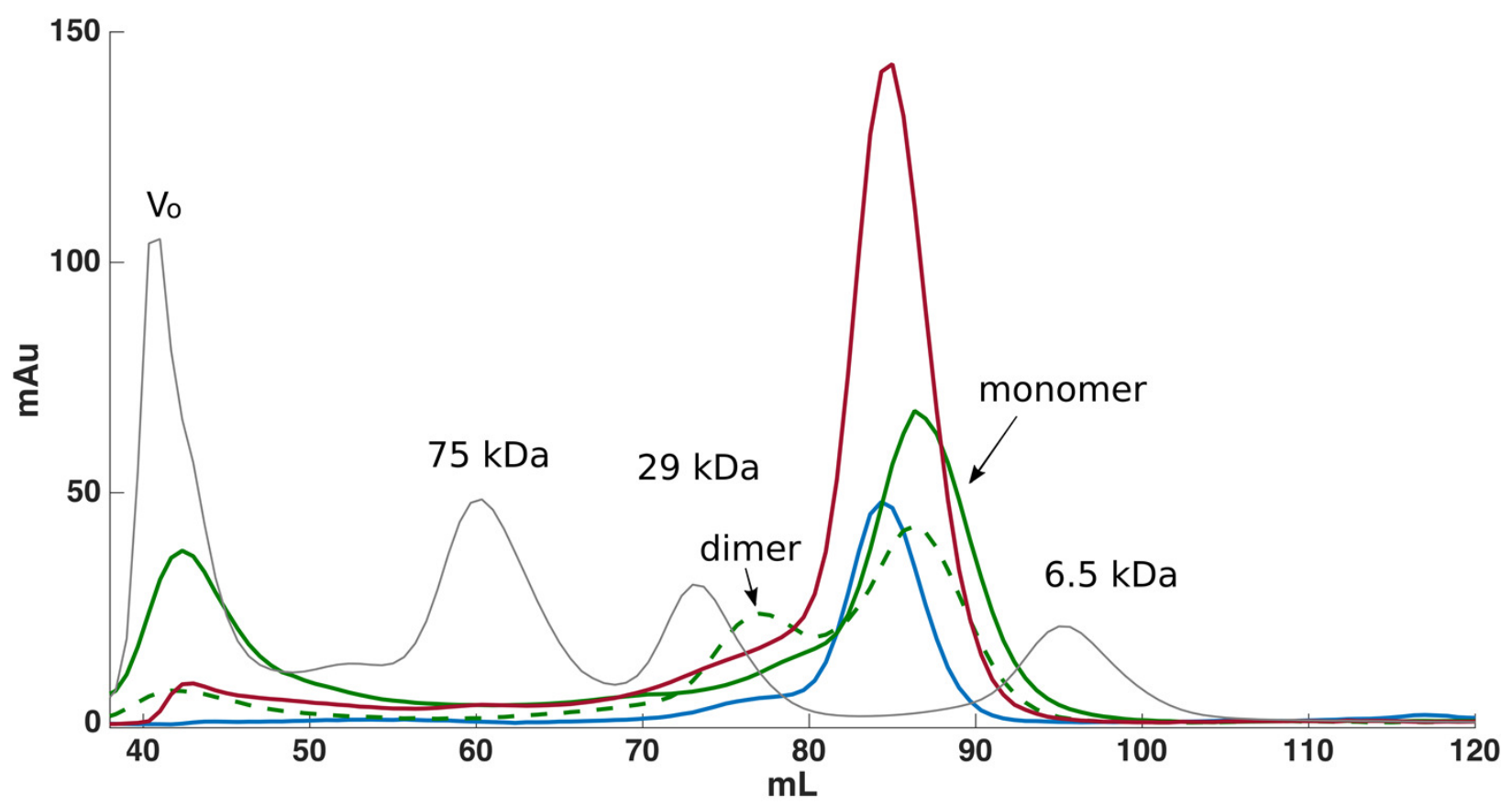

Figure 1. Size exclusion chromatography was used for further purification of FLRT-FnIII samples. Initially purification was attempted without DTT, which resulted in monomeric and dimeric forms of FLRT2-FnIII as seen from the SEC curve (green, dash line). After including DTT the presence of dimeric forms is significantly reduced although a small fraction is still present (green). Subsequent purifications of FLRT1- (blue) and FLRT3-FnIII (red) included DTT, and resulted in similar profile with monomers as the predominant form. Standards in grey (Blue Dextran to determined Void volume, Vo; Conalbumine, $75 \mathrm{kDa}$; Carbonic Anhydrase, $29 \mathrm{kDa}$; Aprotenin, $6.5 \mathrm{kDa}$ ) were used to calibrate the column, and estimate the molecular weights $(\mathrm{Mw})$ of the FnIII domains.

572

573

574

575

576

577

578

579 
580 FIGURE 2

$\mathrm{kDa}$

60

50

40

30

20

15

10

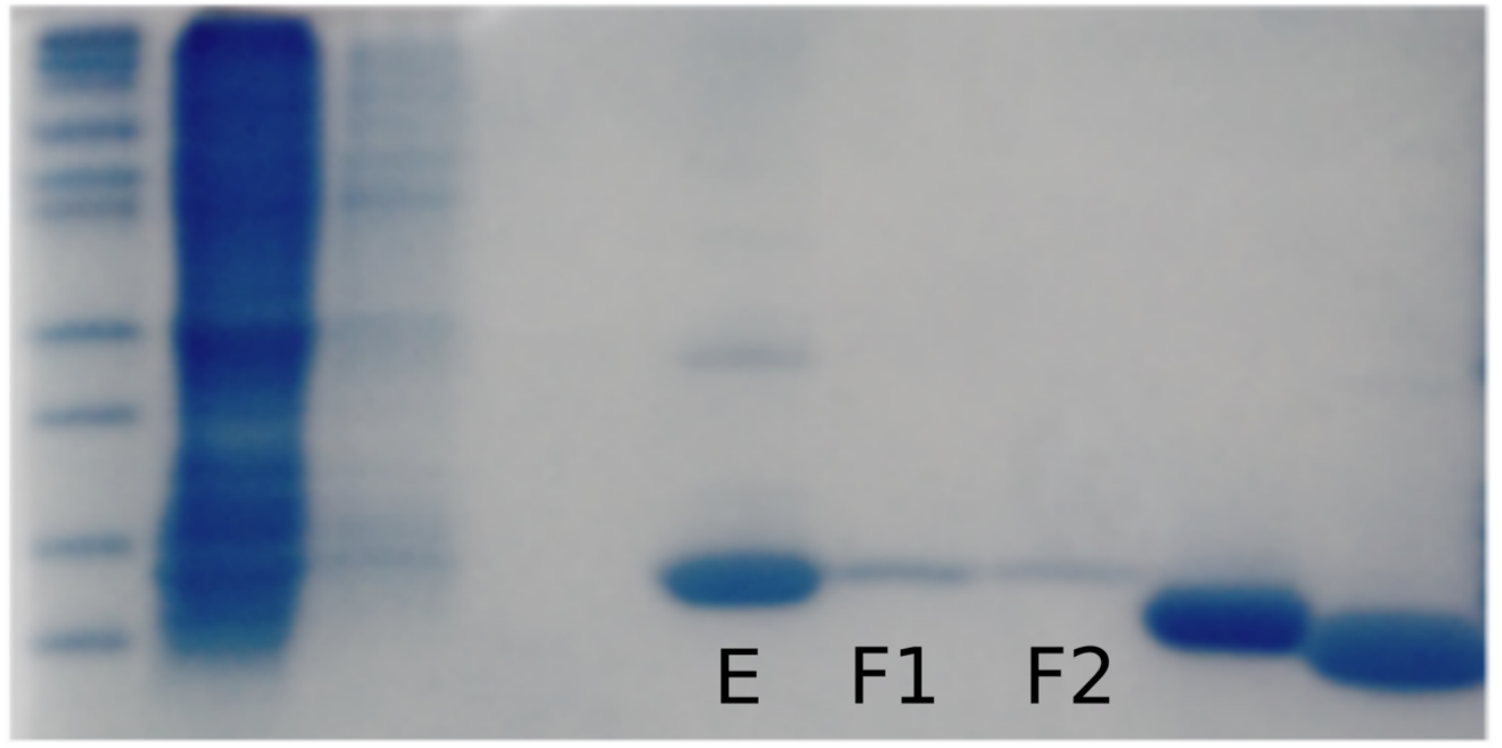

\section{Std FT W G 1Fnlll $\quad$ 3Fnlll 2Fnlll}

581

582

583 Figure 2. SDS-PAGE gel following purification and refolding of FLRT1-FnIII together with 584 purified and concentrated FLRT2-FnIII and FLRT3-FnIII domains. To verify purity and to 585 follow expression, refolding and purification steps, samples were taken at different steps; 586 flow through the nickel-column (FT), wash of immobilized sample (W), application of 587 refolding gradient $(\mathrm{G})$, and elution (E). Also from the SEC purification samples were taken 588 from different fractions (F1 and F2). FLRT2-FnIII and FLRT3-FnIII were purified and 589 refolded following the same protocol. Concentrated samples can be seen next to FLRT1-FnIII 590 purification, showing pure proteins. Markers are also shown (Std). 


\section{FIGURE 3}

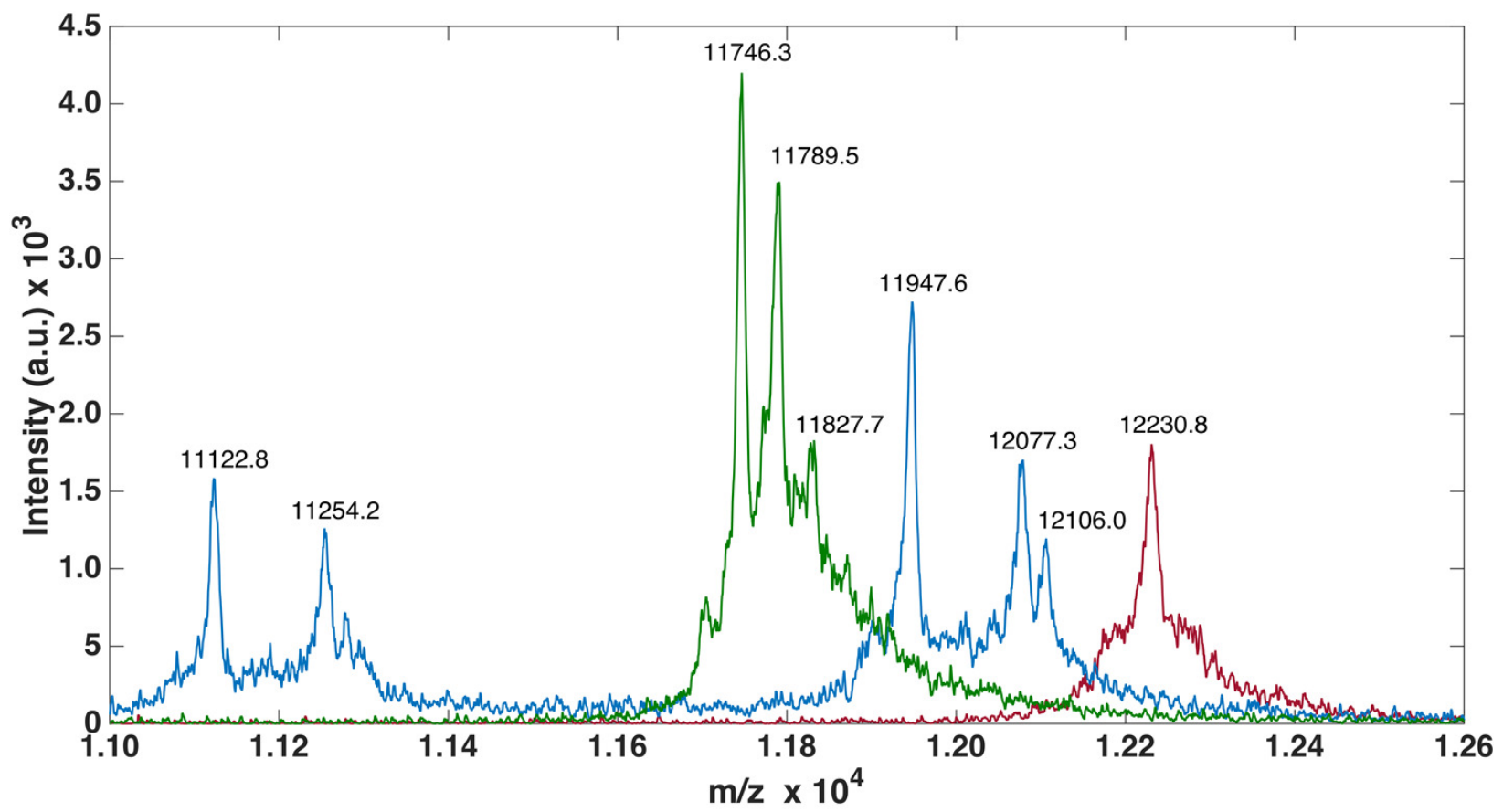

593

594 Figure 3. MALDI-TOF analysis of FLRTs domains. MALDI-TOF was performed to validate 595 purity. FnIII domains from FLRTs were all prone to degradation. For FLRT1 (blue) and FLRT2 596 (green) domains the degradation was more pronounced than for FLRT3 (red).

597

598

599

600

601

602

603

604

605

606

607

608

609

610

611 


\section{FIGURE 4}

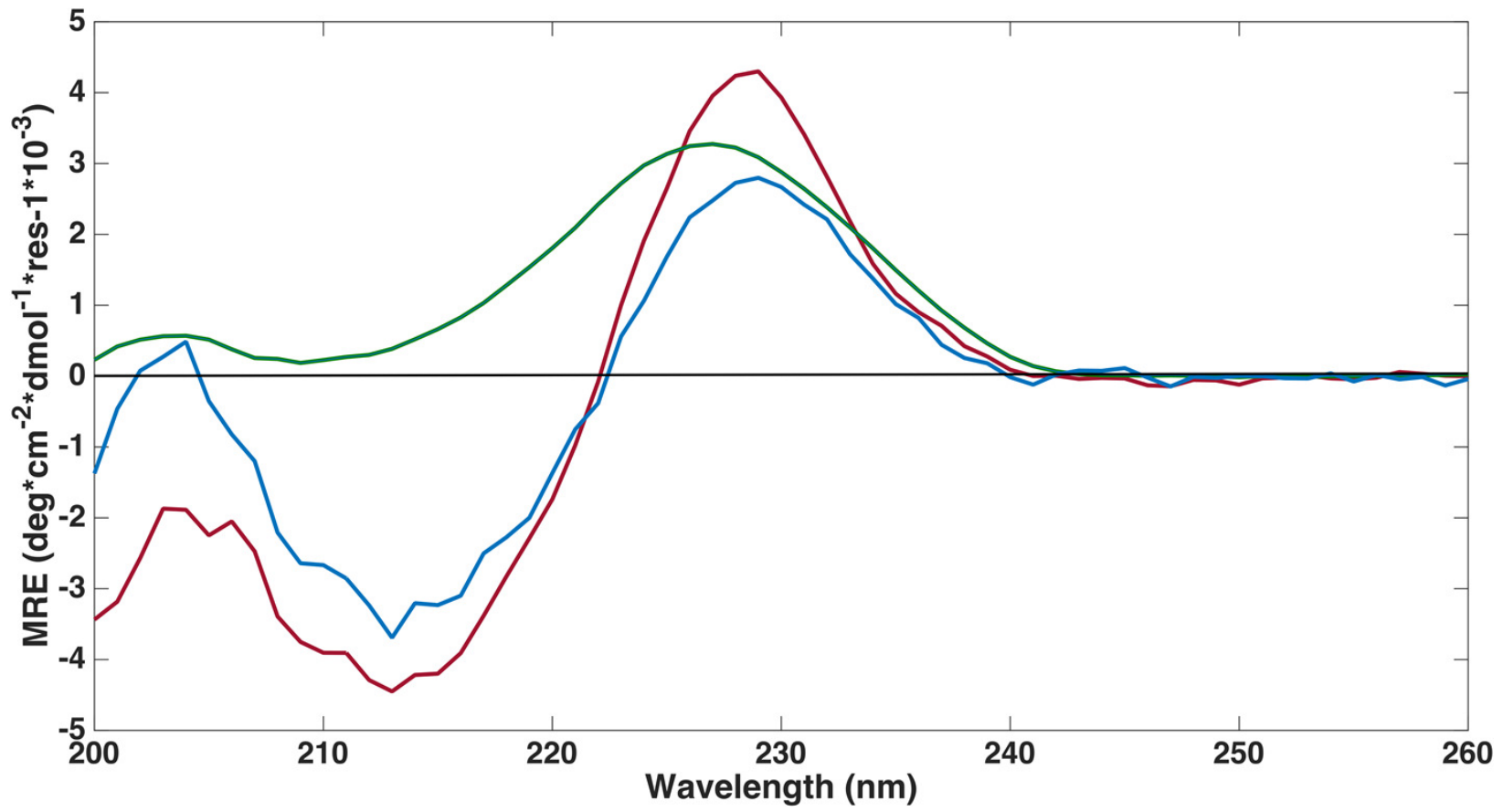

614 Figure 4. Far-UV circular dichroism spectroscopy was performed to assess if proteins were

615 folded. CD spectroscopy experiments were conducted on refolded FLRT-FnIII domains 616 (FLRT1-FnIII (blue), FLRT2-FnIII (green) and FLRT3-FnIII (red)) in PBS buffer. All 617 samples have a positive signal around $230 \mathrm{~nm}$, which is characteristic for fibronectin 618 domains. MRE is mean residue ellipticity.

619

620

621

622

623

624

625 
626

627

FIGURE 5
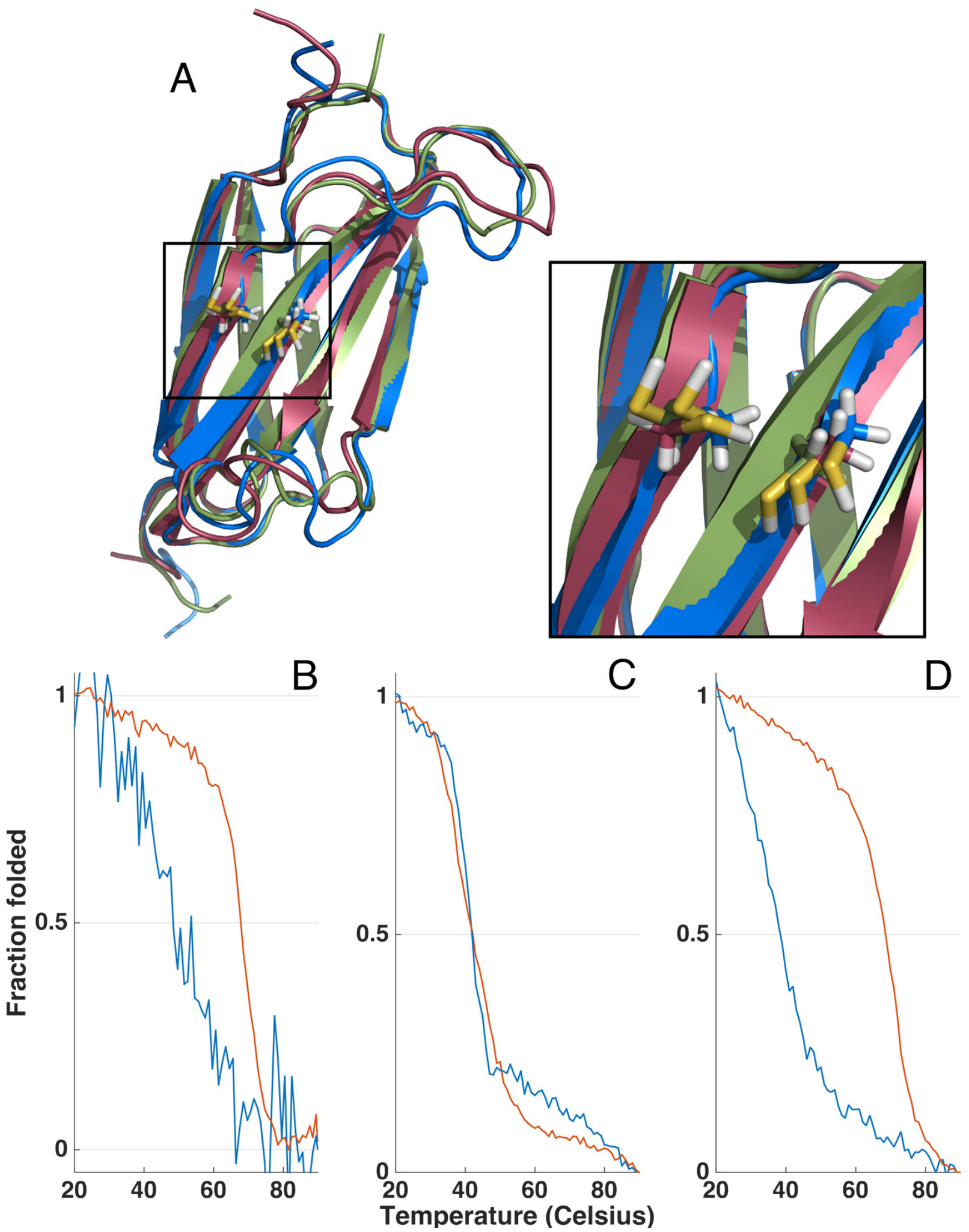

630 
631

632 Figure 5. Homology models and thermal unfolding of the FnIII domains from the FLRT family 633 as monitored by the CD signal at $228.5 \mathrm{~nm}$, normalized to vary between 1 and 0 for the initial 634 and final data point in each series. The I-TASSER server (Yang et al., 2014) was used to predict 635 the topology and the location of the two cysteins located in the FnIII domains of FLRT1 (blue), 636 FLRT2 (green) and FLRT3 (red) (A). In all the I-TASSER modelled FLRT-FnIII domains the 637 two cysteines are located in closed vicinity on each $\beta$ stand (F and G) (A, zoom in). For FLRT1638 FnIII (B) and FLRT3-FnIII (D) there is a large difference in thermal unfolding curves in the 639 presence (blue line) and absence (red line) of reducing agent, DTT. In contrast, thermal 640 unfolding of FLRT2-FnIII is not affected by DTT (C) as for FLRT1-FnIII and FLTR3-FnIII. 